\title{
APPLICATION OF A MEMORY SURFACE MODEL TO PREDICT WHOLE-LIFE SETTLEMENTS OF A SLIDING FOUNDATION
}

\author{
Published in Computers and Geotechnics 88:152-163 \\ https://doi.org/10.1016/j.compge0.2017.03.014
}

\begin{abstract}
Riccardo Corti. Geotechnical Engineer (former PhD Candidate, Department of Civil Engineering, University of Bristol), Arup, 13 Fitzroy Street, W1T 4BQ, London (corresponding author). E-mail: Riccardo.Corti@arup.com
\end{abstract}

Susan M. Gourvenec. Professor, Centre for Offshore Foundation Systems, a node of the ARC Centre of Excellence for Geotechnical Science and Engineering, University of Western Australia, 35 Stirling Highway, Crawley, WA 6009, Australia. E-mail: Susan.Gourvenec@uwa.edu.au

Mark F. Randolph. Professor, Centre for Offshore Foundation Systems, a node of the ARC Centre of Excellence for Geotechnical Science and Engineering, University of Western Australia, 35 Stirling Highway, Crawley, WA 6009, Australia. E-mail: Mark.Randolph@uwa.edu.au

Andrea Diambra. Lecturer, Department of Civil Engineering, Queen’s Building, University of Bristol, Bristol BS8 1TR, UK. E-mail: Andrea.Diambra@bristol.ac.uk

Number of words: 5633 (excluding abstract, tables, notation list and references)

Number of figures: 17 , Number of tables: 3 


\begin{abstract}
In this paper a novel modelling procedure is proposed to estimate whole-life settlements of tolerably mobile sliding foundations. A new kinematic hardening-critical state-state parameter constitutive model, the Memory Surface Hardening model, is implemented in a onedimensional analysis to predict accumulated vertical settlements under drained lateral cyclic loading. The Memory Surface Hardening model performance is compared with the Modified Cam Clay and Severn-Trent Sand models. The Memory Surface Hardening model is adopted to simulate available experimental data from centrifuge tests to predict the settlement of a sliding foundation at the final stable state (i.e. no further volume changes occur).
\end{abstract}

Keywords: Settlement; cyclic loading; offshore engineering; soil modelling; memory surface. 


\section{$1 \quad 1 \quad$ Introduction}

2 Sliding foundations are a novel concept to meet the increasingly challenging demand to limit

3 the footprint of subsea mudmats. In contrast to the traditional paradigm that foundations remain

4 stationary and resist all the applied loads, sliding foundations are designed to move tolerably

5 across the seabed to relieve some of the applied loads, thus requiring a smaller footprint. Sliding

6 displacements are caused, and also limited, by expansion and contraction of attached pipelines

7 ([1], [2], [3]). In general, magnitudes of displacement are sufficient to cause shear failure

8 between the foundation and the soil, where the mobilised ratio of shear stress to normal

9 effective stress is greatest.

10 Subsea mudmats are shallow, mat-style foundations used to support pipeline infrastructure for

11 offshore hydrocarbon developments. Foundation loads derive from the self-weight of the mat, the supported structure and thermal expansion and contraction of the attached pipelines. Increasing operational loads coupled with softer seabeds has resulted in traditional subsea mudmat designs exceeding the installation capacity of pipelaying vessels. The expense of an additional heavy lift vessel on site to install over-sized mudmats can be prohibitive. Sliding foundations offer a potential solution to this impasse ([1], [2], [3]).

Observations of performance of a sliding foundation on soft clay from a programme of centrifuge model tests are reported by Cocjin et al. [2]. The considered sliding mudmat comprised a rectangular rough-based mat of breadth to length aspect ratio of 0.5 and was provided with edge 'skis' to facilitate sliding (rather than overturning that may lead to overstressing of the pipeline connections). A schematic representation of the generalized geometry is presented in Fig. 1, also showing an attached pipeline connection. The tests involved a number of cycles of undrained sliding with intervening periods of consolidation. The model data showed settlement of the mat during each period of consolidation resulting from dissipation of shear induced pore pressures generated during the preceding sliding event. 
The accumulated mat settlements reduced with each slide, ultimately reaching a stable state condition with no further volume change in the soil. This stable state was shown to be equivalent to the drained state [4].

29

This last observation is illustrated in Fig. 2, through an analysis of a strain-imposed cyclic simple shear test under constant total vertical stress conditions using the Modified Cam Clay model [5]. Results compare the stress-volume changes under drained cycles of loading and undrained cycles of loading with intervening periods of consolidation. It is evident that the volumetric behaviours are comparable and the final stable state from the two simulations converge. A further check is performed by comparing the variation of the void ratio with the number of cycles for the performed simulations. The trends are similar, which confirms that the soil response is comparable.

It can therefore be surmised that consideration of drained sliding and associated (drained) volumetric strain is an appropriate approximation for undrained generation and subsequent dissipation of shear induced excess pore pressures. On this assumption, this study investigates the volumetric response of drained lateral cyclic loading of a sliding foundation. Three constitutive models are adopted to estimate vertical settlements over the whole-life of a sliding foundation; predicted results are compared with available data from centrifuge tests performed at the University of Western Australia - Centre for Offshore Foundation Systems (UWACOFS) [2]. 


\subsection{Analysis set up}

The framework of the 1-D analyses considering a sliding mudmat of width $B$ resting on halfspace soil is shown in Fig. 3. The overall soil response under the shearing imposed by the sliding mudmat is computed through a layer-by-layer summation, by dividing the half-space soil domain into $n$ different layers of thickness $h_{i}$, where $i$ is the layering index, up to the depth of influence of the loading imposed by the sliding mudmat. The average response of the individual layer $i$ is computed at a characteristic soil element located at its vertical mid-point, as shown in Fig. 3. Since the large stress gradient in the layers close to the foundation base may affect the accuracy of layer-average based procedure, it is customary to use thinner layering in the uppermost layers. The loading conditions in each layer was approximated using available elastic solutions for vertical and shear load distribution with depth [6], while the soil response was computed assuming the three specific elasto-plastic constitutive models outlined above (and described in detail below). Comparison of the shear stress distribution beneath a surface foundation under horizontal sliding in an elasto-plastic medium determined from a finite element analysis (described in [7]) showed adoption of an elastic shear stress distribution to be an appropriate simplification (Figure 4). Each elasto-plastic model was coded in Matlab and solved incrementally using the fourth order Runge-Kutta numerical integration method.

\subsection{Constitutive soil models}

Three soil constitutive models were used to predict the accumulated settlement during drained lateral cyclic loading: the Modified Cam Clay model (MCC) [5], the Severn-Trent Sand model (STS) [8-9] and the Memory Surface Hardening model (MSH) [10-12], which are represented 
are constructed within the framework of Critical State Soil Mechanics [13,14]. The critical state is modelled as a straight line in the void ratio-mean effective pressure semi-logarithmic plane $\left(e-\ln p^{\prime}\right)$. The deviatoric section of all the yield surfaces follows the shape proposed by Argyris et al. (1974) [15] which avoids the presence of singularities and ensures that the critical state strength varies with the Lode angle. The main differences between the models are: a) the shape of the model surfaces in the $q-p^{\prime}$ plane, which are ellipses for the MCC and wedges for the other two models (STS and MSH), b) the adopted dilatancy rule (defined by $d$ in Fig. 5) and, most importantly, c) the number of model surfaces (defined by their slope $\eta$ in Fig. 5) which increases from the simplest MCC model using only a yield surface, to the most sophisticated MSH model, which postulates the existence of yield, bounding and memory surfaces. The larger number of model surfaces implies an increased complexity of the employed hardening rule which can allow a more accurate simulation of the non-linear stress and density dependent behaviour of soil as well as the influence of past stress history. This last aspect is expected to be the most important to accurately predict the cyclic response of the sliding mudmat in the following analyses. A brief description of each of the three soil models is provided in the following alongside schematic representations in Fig. 5. The most fundamental mathematical relationships are also reported in Table 1. The full mathematical formulation for each model can be found in the relevant referenced literature as referenced below.

Modified Cam Clay (MCC) model [5]: An elasto-plastic model that assumes an elliptical yield locus that passes through the origin of the stress plane and bounds soil elastic states (Fig. 5a). The size of the yield locus is governed by the parameter $p_{c}$. Under elasto-plastic loading conditions, the yield locus evolves preserving its shape and its intersection with the origin of 
the stress state axis, with the consequent variation in size of the elastic region. An associative flow rule is assumed.

Severn-Trent Sand (STS) model [8,9]: A kinematic hardening - bounding surface elasto-plastic constitutive model. A purely elastic region is bounded by the yield surface $\left(f_{Y}\right)$ which is an open wedge with its apex at the origin of the $q-p$ ' stress axes (Fig. 5b) and moves in the stress space (kinematic hardening) under shearing. The bounding surface $\left(f_{B}\right)$ represents the current soil strength and its size is influenced by the current soil density through the state parameter [16]. Based on the bounding surface theory $[17,18]$, plastic soil stiffness is assumed to depend on the distance between the current stress state $\sigma$ and the conjugate one on the bounding surface $\boldsymbol{\sigma}^{\boldsymbol{B}}$, as shown in Fig. 5 (b) and in Table 1. Upon unloading-reloading, the soil response is initially fully elastic inside the yield surface but elasto-plastic conditions are invoked when the opposite boundary of the yield locus is reached.

Memory Surface Hardening (MSH) model [10-12]: A recently developed constitutive model based on an extension of the STS model by introducing an additional surface, the memory surface $\left(f_{M}\right)$, which retains information of the recent stress history (Fig. $5 c$ ). The memory surface bounds a region of stress states that the soil has already experienced. When the current stress state lies inside the memory surface, the memory surface $f_{M}$ acts as an additional bounding surface so that the plastic soil modulus is governed by an additional hardening term depending on the distance between the current stress $(\sigma)$ state and its projection on the memory surface $\left(\sigma^{M}\right)$. This results in a stiffer soil behaviour during repeated loading compared with virgin loading conditions. The memory surface can evolve in size and translate in the stress space according to three rules: 
124 Rule 1: Changes in size of the memory surface are linked to plastic strains;

125

Rule 2: The current stress state must always lie inside or on the boundary of the memory surface;

Rule 3: The yield locus must always be enclosed within the memory surface.

Table 1: Summary of functions used in the selected constitutive models

\begin{tabular}{|l|c|c|c|}
\hline & $\begin{array}{c}\text { Modified Cam Clay } \\
{[5]}\end{array}$ & $\begin{array}{c}\text { Severn-Trent Sand } \\
{[\mathbf{8 , 9 ]}}\end{array}$ & $\begin{array}{c}\text { Memory Surface Hardening } \\
\text { [10, 12] }\end{array}$ \\
\hline $\begin{array}{l}\text { Yield } \\
\text { surface }\end{array}$ & $f_{Y}=\frac{q^{2}}{M^{2}}+p^{\prime}\left(p^{\prime}-p_{c}\right)$ & $f_{Y}=q-\eta^{Y} p^{\prime}$ & $f_{Y}=q-\eta^{Y} p^{\prime}$ \\
\hline $\begin{array}{l}\text { Bounding } \\
\text { surface }\end{array}$ & --- & $f_{B}=q-\eta^{B} p^{\prime}$ & $f_{B}=q-\eta^{B} p^{\prime}$ \\
\hline $\begin{array}{l}\text { Memory } \\
\text { surface }\end{array}$ & --- & --- & $f_{M}=\eta^{M} p^{\prime}$ \\
\hline Flow rule & $d=\frac{M^{2}-\eta^{2}}{2 \eta}$ & $d=A_{d}\left[\left(1+k_{d}\right) M-\eta\right]$ & $d=A_{d}\left[\left(1+k_{d}\right) M-\eta\right]$ \\
\hline $\begin{array}{l}\text { Hardening } \\
\text { modulus }\end{array}$ & $H=p^{\prime}\left(\frac{v p^{\prime}{ }_{c}}{\lambda-\kappa}\right)\left(2 p^{\prime}-p^{\prime}{ }_{c}\right)$ & $H=\frac{b^{2}}{b_{\max } \beta}$ & $H=\frac{b^{2}}{b_{\max } \beta} \exp ^{\left[\frac{\mu(1-k \psi) b^{M}}{b}\left(\frac{p^{\prime}}{p_{r e f}}\right)^{0.5}\right]}$ \\
\hline
\end{tabular}

The hardening modulus $H$ presented in Table 1 is introduced to calculate the elasto-plastic stiffness matrix. Following [8,9], this is calculated as

$$
\boldsymbol{D}^{e p}=\boldsymbol{D}^{e}-\frac{\boldsymbol{D}^{e} \boldsymbol{m} \boldsymbol{n}^{T} \boldsymbol{D}^{e}}{\boldsymbol{n}^{T} \boldsymbol{D}^{e} \boldsymbol{m}+H}
$$

Where $\boldsymbol{D}^{e}$ is the elastic stiffness matrix, $\boldsymbol{n}$ is the normal outwards from the yield surface and $\boldsymbol{m}$ is the normal outwards from the plastic potential surface. An exhaustive description of the terms is provided in [8,9] and [12]. It should be noted the slightly different formulation of the hardening modulus of the Memory Surface Hardening model from the one defined in [10-12], where the dependence on the ratio $\left(p^{\prime} / p_{\text {ref }}\right)^{0.5}$ is inspired by the previous work from Hardin and 
Black [19] and it accounts for the influence of the effective mean stress. The quantity $b$ represents the distance between the current stress state $(\sigma)$ and the image stress on the bounding surface $\left(\boldsymbol{\sigma}^{\boldsymbol{B}}\right)$ with $b_{\max }$ its maximum value, the quantity $b^{M}$ is the distance between the current stress state $(\sigma)$ and the image on the memory surface $\left(\sigma^{M}\right), \psi$ is the state parameter, $p^{\prime}$ is the effective mean pressure, $p_{\text {ref }}$ is a reference pressure $\left(p_{\text {ref }}=100 \mathrm{kPa}\right)$ and $\mu$ and $\beta$ are two constitutive parameters. The constitutive parameters $\beta$ and $\mu$ are quite relevant for this application because they govern the magnitude of the accumulated strains during the first slide and under cyclic loading conditions, respectively. It should be noted that by imposing the parameter $\mu=0$, the hardening modulus of the STS is re-established.

\section{Calibration of the constitutive parameters}

Summary and description of the constitutive parameters for the three models considered are provided in Table 2. The number of constitutive parameters required by the models is proportional to their complexity - 5, 10 and 12 for the MCC, STS and MSH respectively - but, as shown in Table 2, the fundamental parameters are shared and the increased complexity is reflected only in the use of additional parameters. For consistency in the following analysis, the shared constitutive parameters must also assume the same value for the different models employed. The calibrated numerical values of the model parameters are also provided in Table

2. The first five constitutive parameters in Table 2 are shared by all three models and have been calibrated in accordance with Stewart [20] and Acosta-Martinez and Gourvenec [21], who used the MCC model to simulate the behaviour of exactly the same kaolin clay material used in the centrifuge model tests [2] back-analysed in this paper. Thus, only the calibration procedure for the other constitutive parameters (no. 6 to 12 in Table 2) relative to the STS and MSH models is presented in this section. The constitutive parameters of the STS and MSH models have been calibrated against an available drained cyclic triaxial test on speswhite kaolin clay [22]. 
Information on the mechanical properties of this material can be found in [23] and shows the kaolin is similar to that used in the centrifuge tests [2]. The shared constitutive parameters between the STS and MSH (No. 6 to 10 in Table 2) have been calibrated by fitting the initial virgin behaviour while the constitutive parameter $\mu$ has been calibrated by fitting the experimental data under the subsequent cyclic conditions. The damage parameter $\varsigma$ of the MSH model governing an eventual damage of the memory surface $\left(f_{M}\right)$ upon dilation is not included because it is not relevant for the very soft soil conditions simulated in this study. The model calibration against an available cyclic drained triaxial test is provided in Fig. 6.

Table 2: Constitutive parameters description.

\begin{tabular}{|c|c|c|c|c|c|}
\hline \multicolumn{3}{|c|}{ Constitutive model } & No. & Parameter & \\
\hline \multirow{7}{*}{$\begin{array}{c}\text { Memory } \\
\text { Surface } \\
\text { Hardening }\end{array}$} & \multirow{5}{*}{ Severn-Trent } & \multirow{5}{*}{$\begin{array}{c}\text { Modified Cam } \\
\text { Clay model }\end{array}$} & 1 & $\kappa$ & 0.044 \\
\hline & & & 2 & $M_{c v}$ & $\begin{array}{c}0.92 \\
\left(\phi^{\prime}=23.5^{\circ}\right)\end{array}$ \\
\hline & & & 3 & $e_{C S L}$ & 2.14 \\
\hline & & & 4 & $\lambda$ & 0.205 \\
\hline & & & 5 & $v$ & 0.3 \\
\hline & & & 6 & $R$ & 0.05 \\
\hline & & & 7 & $\beta$ & 0.017 \\
\hline \multirow{5}{*}{ model } & & & 8 & $k^{*}$ & 1.5 \\
\hline & & & 9 & $A_{d}$ & 2.5 \\
\hline & & & 10 & $k_{d}$ & 0 \\
\hline & & & 11 & $\mu$ & 11.5 \\
\hline & & & 12 & $\varsigma$ & n. $r$. \\
\hline
\end{tabular}




\section{$4 \quad$ Analysis procedure}

178

$$
\sigma_{h 0}^{\prime}=K_{0} \sigma^{\prime}{ }_{v 0}
$$


where $K_{0}$ is the lateral earth pressure coefficient given by Jaky's formula [24]:

203

204

$K_{0}=1-\sin \phi^{\prime}$

205

206

with the critical state friction angle $\phi^{\prime}$ as assumed in Table 2.

207

The initial void ratio is estimated using the MCC model formulation and imposing normally consolidated conditions:

209

$210 e_{0}=e_{C S L}-(\lambda-\kappa) \ln \frac{p_{c 0}^{\prime}}{2}-\kappa \ln p_{0}^{\prime}$

211

where $p_{0}^{\prime}$ is the current in situ effective mean pressure determined using $\sigma_{v 0}^{\prime}$ and $\sigma_{h 0}^{\prime}$. The quantity $p^{\prime}{ }_{c 0}$ is the pre-consolidation pressure defining the size of the MCC yield locus that can

214 be derived using the formulation of the yield locus for the MCC model:

$$
p_{c 0}^{\prime}=p_{0}^{\prime}+\frac{q_{0}^{2}}{p_{0}^{\prime} M^{2}}
$$

where $q_{0}=\sigma_{v 0}^{\prime}-\sigma_{h 0}^{\prime}$ is the deviator stress. The estimated initial in situ vertical and horizontal effective stress profiles, $\sigma_{v 0}^{\prime}$ and $\sigma_{h 0}^{\prime}$, and void ratio profile $e$ with depth $z$ are shown in Fig. 7ab respectively.

\subsection{Foundation installation stage}

The change in vertical effective stress $\Delta \sigma_{v}^{\prime}$ with depth $z$ resulting from placement of the foundation load is approximated with the elastic solution of Poulos \& Davis (1974) [6]. The stress increase below the centre of a rectangular foundation induced by a uniform vertical pressure $q_{o p}$ can be expressed as: 
$\Delta \sigma_{v}^{\prime}=2 \frac{q_{o p}}{\pi}\left[\frac{B}{2 A}\left(1+\frac{z^{2}}{2 A^{2}}\right) \operatorname{atan} \frac{L}{2 A}+\frac{L}{2 D}\left(1+\frac{z^{2}}{2 D^{2}}\right) \operatorname{atan} \frac{B}{2 D}+\frac{B L Z^{2}}{8 C^{2}}\left(\frac{1}{A^{2}}+\frac{1}{D^{2}}\right)\right]$

230 where $A, B, C, D, L$ and $z$ are geometrical distances represented schematically in Fig. 8.

231 A schematic representation of the normalized vertical stress change $\Delta \sigma_{v}^{\prime} / q_{o p}$ with depth $z$ is represented in Fig. 9a. At this stage, the value of the lateral earth pressure coefficient assumed in Eq. (4) was compared to the stress ratio calculated assuming a horizontal stress change from the elastic solution of Poulos \& Davis (1974) [6]. At a normalized depth z/B of 0.1, a difference of $15 \%$ was found, with the $K_{0}$ value calculated in Eq. (4) being larger than the horizontal stress ratio calculated using the elastic solution. This difference reduced to $10 \%$ at a normalised depth $\mathrm{z} / \mathrm{B}=0.3$.

The magnitude of the foundation vertical pressure $q_{o p}$ is a variable (input) parameter and can be defined as a portion of the available undrained bearing capacity $q_{u}$. In field conditions for subsea mudmats on soft clays, the ratio $q_{o p} / q_{u}$ is generally between 0.3 and 0.5 [2]. For consistency with the centrifuge tests [2], the foundation vertical pressure $q_{o p}$ is set to satisfy the following condition:

$q_{o p}=0.3 q_{u}$

To ensure consistency between the calculated bearing capacity and the constitutive models, the undrained shear strength profile is determined from the assumed elastic and critical state soil properties. Following the procedure outlined in [25], it is possible to determine the undrained shear strength profile using the input parameters of the MCC model as shown in Eqs (9) to 250 $(11)$. 
$251 \frac{s_{u}}{\sigma^{\prime} v_{0}}=g(\theta) \cos \theta \frac{1+2 K_{0}}{3}\left[\frac{\left(a^{2}+1\right)}{2}\right]^{1-\left(\frac{\kappa}{\lambda}\right)}$

252 where

$253 g(\theta)=\frac{\sin \phi^{\prime}}{\cos \theta+\left(\frac{1}{\sqrt{3}}\right) \sin \phi^{\prime} \sin \theta}$

254 and

$255 \quad a=\frac{\sqrt{3}\left(1-K_{0}\right)}{g\left(-30^{\circ}\right)\left(1+2 K_{0}\right)}$

256 The symbol $\theta$ is the Lode's angle, taken as 0 to represent plane strain shear strength. The undrained shear strength profile is calculated by assuming the same undrained shear strength

258 at the surface $s_{u m}=0.52 \mathrm{kPa}$ as that measured in situ; to do this, an extra overburden stress of $1.85 \mathrm{kPa}$ is applied in situ. An undrained shear strength gradient $k=1.70 \mathrm{kPa} / \mathrm{m}$ is derived from the critical state properties, giving a dimensionless strength heterogeneity coefficient $\mathrm{kB} / \mathrm{sum}_{\mathrm{um}}$ 16. Bearing capacity factors for rectangular foundations are available for soil strength heterogeneity in the range $0 \leq k B / s_{u m} \leq 10$ [26], while solutions exist for the appropriate $k B / s_{u m}$ but assuming plane strain geometry [27]. The outcome from extrapolating a bearing capacity factor for the rectangular foundation geometry to the appropriate $k B / s_{u m}$ for this example is similar to the plane strain solution (approximately 10\% lower). For this simulation the operative bearing pressure is taken as

$q_{o p}=0.3 N_{c V} s_{u m}=0.3(15.85)(0.52 \mathrm{kPa})=0.3(8.25 \mathrm{kPa})=2.48 \mathrm{kPa}$

By introducing the above value of $q_{o p}$ in Eq. (7), the increase of vertical stress $\Delta \sigma_{v}^{\prime}$ for the characteristic points of each layer $i$ can be determined. Assuming $K_{0}$ compression conditions, the Modified Cam Clay model has been employed to determine the vertical strain $\varepsilon_{v}^{i}$ and the void ratio $e^{i}$ at each characteristic point. The overall vertical settlement of the soil can be 
$\tau=2 \frac{\tau_{\max }}{\pi}\left[\operatorname{atan} \frac{B}{2 z}-\frac{z}{D} \operatorname{atan} \frac{B}{2 D}+\frac{B}{2 z}\left(\frac{B^{2}}{4 A^{2}}-\frac{B^{2}+L^{2}}{4 C^{2}}\right)\right]$

$$
\delta_{v}=\sum_{i=1}^{n} \varepsilon_{v}^{i} h^{i}
$$

\subsection{Foundation sliding stage} foundation operative vertical stress $q_{o p}$ :

$$
\tau_{\text {max }}=q_{o p} \tan \phi^{\prime}=1.08 \mathrm{kPa}
$$

Under operational conditions, the foundation is subjected to forward and backward cyclic sliding. The concept of a sliding mudmat is such that the foundation is free to exceed by far the displacement needed to mobilize the limiting soil-foundation shear resistance to sliding, $\tau_{\text {max. }}$. The limiting soil-foundation shear resistance, which represents the (average) shear stress cyclic amplitude which the sliding foundation is subjected to, is found as a proportion of the

where the friction between the mudmat and the soil is a function of the friction angle tan $\phi^{\prime}$, as was also detected in the experimental tests [2]. The shear stress distribution with depth during full sliding can be predicted using the idealized elastic solution for a foundation on a semiinfinite soil mass as a function of the shear stress on the surface $\tau_{\max }[6]$ :

295

The distribution of shear stress $\tau$ normalized by the interfacial shear stress $\tau_{\text {max }}$ with depth during sliding is shown in Fig. 9b. The horizontal cyclic loading acting on the foundation has been reproduced considering equivalent simple shear conditions; the shear stress cyclic 
amplitude $\Delta \tau^{c y c}$ has been derived from Eq. (15) by assuming $\Delta \tau^{c y c}= \pm \tau$, where the sign \pm is implemented to include the direction of the loading cycles (forward or backwards). The simulations of the foundation sliding stage have been performed separately for the three constitutive models (MCC, STS and MSH) in order to investigate their capabilities. A total of 40 back-and-forth cycles have been imposed in order to simulate the long-term behaviour of sliding foundations. The overall foundation settlement for this stage is determined using Eq. (13) above.

\section{$5 \quad$ Analysis results}

\subsection{Model performances}

Comparison of the predicted foundation vertical settlements normalised by the width of the foundation $\left(\delta_{v} / B\right)$ during the 40 imposed shearing cycles is presented in Fig. 10a for the MCC model, Fig. 10b the STS model and Fig. 10c for the MSH model. A direct comparison of evolution of the vertical settlements with number of loading cycles is also presented in Fig. 10d. The predicted trends and magnitude of settlements differ considerably among these models. The MCC model predicts a normalized vertical displacement $\delta_{v} / B=9 \cdot 10^{-4}$ at the stable state, which is reached at the end of the very first shearing cycle with no further densification occurring with subsequent cycles since the load path lies within the now expanded yield surface (Fig. 10a). On the contrary the STS model predicts a much larger normalised vertical displacement, $\delta_{v} / B=1.5 \cdot 10^{-1}$ after 40 cycles (Fig. 10b). In this case, a stable state is not reached as the model does not accurately capture the rate of plastic strain hardening. The prediction of the MSH model lies between the MCC and STS model predictions outlined above, suggesting a normalized vertical displacement $\delta_{v} / B=4.4 \cdot 10^{-2}$ after 40 cycles which is gradually reached during the cyclic loading sequence (Fig. 10c). Settlement measured in the centrifuge test [2] is 
324 also reported in Fig. 10d for comparison with the three computations performed. It is clear that while the MSH model predicts qualitatively and quantitatively the closest behaviour to the experimental observation, the final vertical displacement of the foundation is slightly underestimated. This may be the result of limitations in the calibration exercise performed for experimental results on soil samples tested under different loading conditions (triaxial compression instead of simple shear) and under much larger confining pressure (up to two orders of magnitude).

Fig. 11 shows a comparison among the predictions of the three models for the settlement during the first slide. The normalized settlement predicted by the MCC model is the lowest among the models, which follows directly from the nature of the model (an isotropic hardening model). Moreover, the development of the vertical settlements is interrupted by the initial expansion of the yield surface, which occurs at $N_{c y c}=0.5$ (representing the first half of the foundation slide). The vertical settlements predicted by the STS and MSH models at $N_{c y c}=0.5$ are similar and the small difference is due to the slightly different hardening rule implemented in the models. During the first half loading cycle the soil stiffness in the two models is governed by the constitutive parameter $\beta$. At $N_{c y c}=1$, the difference in the simulated vertical settlement increases as the dependence on the memory surface in the MSH model is activated leading to an increase in the plastic soil stiffness which inevitably reduces the magnitude of predicted vertical settlements. The plastic soil stiffness in the MSH model is now governed by the constitutive parameters $\beta$ and $\mu$.

The difference between the three computations can be understood by analysing the predicted behaviours (Fig. 12), stress paths and evolutions of the yield surfaces (Fig. 13), and void ratio changes (Fig. 14) for the shallowest soil element considered. This soil element is indeed that subjected to the largest shear stress cycles and in turn shows the largest vertical strains. 
348 The observed immediate achievement of the stable state for the MCC model is the result of the isotropic hardening expansion of the yield surface upon the first shearing cycle and the resulting fully elastic behaviour during the following shearing cycles imposed within the purely elastic region bounded by the yield surface (Fig. 12a and Fig. 13a). As expected no changes in void ratio are recorded after the first cycle, as shown in Fig. 14a.

The very large settlements predicted using the STS model (Fig. 12b) are the result of the imposed kinematic hardening to the yield surface and the lack of any mechanism to record that the soil has been subjected to the same shearing conditions in previous cycles. In fact, during cyclic shearing, the yield surface moves up and down kinematically as shown in Fig. 13b and the only difference between subsequent cycles is a slight expansion of the bounding surface caused by the progressive soil densification. The plastic soil stiffness, which is affected by the distance between the current stress state and the image stress on the bounding surface (the quantity $b$ in Fig. 5b), only slightly increases during cyclic shearing and a stable state cannot be reached. Actually, the soil continues to compress indefinitely to reach unreasonable values such as a vertical strain $\varepsilon_{v}=1.00$. The soil would continue to compress unrealistically if further cyclic shearing were to be imposed.

The response of the MSH model lies in between the MCC and STS model responses as shown by the stress-strain behaviour in Fig. 12c. For this model, the yield surface still moves up and down kinematically as shown for the STS model in Fig. 13b, but the introduction of an evolving memory surface (shown in Fig. 13c) allows the progressive stiffening during repeated loading to be captured. One can interpret the memory surface as a record of the current fabric of the soil, describing the range of stresses that can be imposed without major disruptions to particle arrangement. Thus, the memory surface bounds a region of increased stiffness and its progressive increase during cyclic shearing allows a progressive increase of the plastic soil stiffness. In this case a stable state is gradually reached with increased number of cycles. 
373 Similarly to the STS model, the MSH model predicts a compression of the soil element to a

374 void ratio well below the critical state line (Fig. 14c) but the progressive soil stiffening prevents

375 the soil from reaching inadmissible states. The independency of the soil volumetric response

376 from the corresponding critical state value during cyclic loading has been confirmed 377 experimentally by several studies on granular soils (e.g. [28-29]). It should also be noted that 378 the response of the STS and MSH model during the first shearing cycle are similar and the 379 additional memory surface of the MSH model affects only the soil behaviour under unloading-

380

\subsection{Sensitivity analysis of MSH model hardening parameter, $\mu$}

In this section a numerical exercise is provided to assess the sensitivity of the MSH model parameter, $\mu$. This parameter affects the magnitude of the soil hardening modulus, thus of soil stiffness, when the stress state lies within the memory surface. The larger the parameter, the stiffer the soil response under cyclic loading but the slower the expansion of the memory surface during progressive cycles. If this parameter is set to zero the STS model is recovered. Fig. 15 indicates the effect of varying the constitutive parameter $\mu$, introduced in Eq. (1), and shows that the predicted vertical settlement at the stable state increases by decreasing the value of the parameter $\mu$. The results from the three simulations are compared directly in Fig. 15d where the accumulated vertical displacements are plotted against the number of cycles; the final displacement measured experimentally by Cocjin et al. [2] is also shown and it seems that 
a value of the parameter $\mu=8$ provides a good fit to the final displacement at the stable state. It should be noted that the value that fits the experimental data $(\mu=8)$ is quite close to that obtained from the model calibration exercise $(\mu=11.5)$.

The foundation set-down settlement is well reproduced by the numerical analysis; experimentally the measured settlement is $0.081 \mathrm{~m}$ while the calculated settlement from the simulation is $0.077 \mathrm{~m}$; the foundation set-down displacement is calculated using the MCC model and assuming $K_{0}$-consolidation conditions.

Using the best-fit simulation to the experimental data it is possible to analyse the predicted trend of void ratio and settlement with depth. Fig. 16a presents the profile of void ratio with normalized depth predicted at the end of the three stages (geostatic, foundation set-down and cyclic shearing), while Fig. $16 \mathrm{~b}$ presents the trends of displacement $\delta_{v}$ normalised by the width of the foundation $B$ for each loading stage. From Fig. 16b, it is clear that while the foundation set-down induces less settlement in the soil than the cyclic shearing stage, it affects layers to a greater depth. The foundation set-down stage affects deeper soil layers and this is confirmed by the percentage of vertical displacements (72\%) occurring within a depth of $0.5 B$. By contrast, the cyclic shearing stage appears to be a very superficial mechanism which just affects the soil to a maximum depth of $0.5 B$; in fact almost $100 \%$ of vertical settlements take place in the top $3 \mathrm{~m}$ of soil $(0.6 B)$. These findings are related to the stress distribution from the assumed elastic solution for the foundation set-down stage (Fig. 9a) and the sliding stage (Fig. 9b). The zone of influence of horizontal cyclic loading is predicted to be limited to a depth of approximately half the foundation breadth, in good agreement with the experimental results [2].

\section{Parametric study}

421 In this section a parametric study is presented to assess the effect on whole-life settlement of 422 sliding foundations after 40 lateral loading cycles as a function of soil strength heterogeneity 
and foundation aspect ratio. The simulations were undertaken using the Memory Surface

424 Hardening model calibrated using the input values given in Table 2, but with the best-fit

425 hardening value presented above $(\mu=8)$. The strength properties, overburden stresses and

426 foundation weights considered in the parametric study are summarised in Table 3. The soil

427 strength properties were obtained using Eqs. (9) to (11), imposing the Modified Cam-Clay

428 constitutive parameters presented in Table 2 and changing the overburden stress. Figure 17 (a)

429 shows the long term normalised foundation settlement with the number of cycles of sliding on

430 soils with different undrained shear strength profiles. Shear strength profiles of $k B / s_{u m}=0,20$

431 and 100 were assumed in this assessment. The vertical displacement after 40 cycles seem rather

432 comparable for a shear strength profile of $k B / s_{u m}=20$ and 100 , where after 40 cycles a

433 normalised displacement of 0.058 and 0.055 is calculated. The normalised displacement for a

434 soil strength $\mathrm{kB} / \mathrm{s}_{u m}=0$ is rather limited as a consequence of the large overburden stress, which

435 implies significant soil compression during the foundation set down stage, as shown in Table

436 3. Figure 17 (b) shows the accumulated normalised vertical displacement of sliding foundations

437 with different aspect ratio $B / L$. The simulations show that the accumulated normalised

438 displacement increases as the aspect ratio $B / L$ decreases.

Table 3: Soil parameters for parametric study of Figure 17 (a)

\begin{tabular}{|c|c|c|c|c|}
\hline $\boldsymbol{k B} / \boldsymbol{s}_{\text {um }}$ & $\boldsymbol{s}_{\text {um }}(\mathbf{k P a})$ & $\boldsymbol{k}(\mathbf{k P a} / \mathbf{m})$ & $\begin{array}{c}\text { Overburden } \\
\text { stress, } \boldsymbol{\Delta} \boldsymbol{\sigma}^{\prime}{ }_{\boldsymbol{v}}(\mathbf{k P a})\end{array}$ & $\boldsymbol{q}_{\text {op }}(\mathbf{k P a})$ \\
\hline 0 & 112 & 2.2 & 400 & 195 \\
\hline 20 & 0.42 & 1.7 & 1.50 & 2.18 \\
\hline 100 & 0.08 & 1.67 & 0.30 & 1.27 \\
\hline
\end{tabular}

440 
442 In this paper a numerical procedure has been proposed to estimate the whole-life settlements

443 of tolerably mobile subsea foundations under cycles of horizontal shearing. The approach is

444 based on the validated assumption that a drained soil response is an appropriate proxy for 445 alternating stages of undrained sliding and consolidation.

446 A one-dimensional model was developed to represent stress and volume changes beneath a mat 447 foundation.

448 Three different constitutive models were used to simulate the soil response to the whole life 449 operation of a sliding foundation: the Modified Cam Clay model, the Severn-Trent Sand model 450 and the Memory Surface Hardening model. Initially the models were calibrated against a 451 drained cyclic triaxial test available in the literature. The Modified Cam Clay model was 452 adopted to simulate the soil consolidation and the foundation set-down stages, assuming $K_{0^{-}}$ consolidation stress path. The foundation sliding stage was then performed by simulating 40cycles of simple shear; the three constitutive models were adopted to calculate the vertical settlements of the foundation. The Severn-Trent Sand model struggled to reproduce the typical stable state that is observed in soils under cyclic loading conditions while the Modified Cam 457 Clay model predicted just elastic strains under drained cyclic loading. The Memory Surface Hardening model was able to reproduce both plastic deformations under cyclic loading and an approach to the stable state.

460 The following aspects were observed:

461

- $\quad$ The vertical displacement related to the foundation set-down stage could be well reproduced by the Modified Cam Clay Model. 
- $\quad$ The maximum density condition (at the steady state) could be well predicted by the

Memory Surface Hardening model but not the Modified Cam Clay Model and Severn Trent Sand models.

- $\quad$ The numerical analysis confirmed that the foundation sliding mechanisms influences just the near surface soil.

469

The Memory Surface Hardening model was demonstrated to successfully calculate the vertical settlements of a sliding foundation on normally consolidated kaolin clay when compared with results from an available centrifuge test. The model applicability should be investigated further for different foundation and soil conditions before being applied outside the conditions considered in this study. Nonetheless, the study has indicated that this class of model has the potential to capture the essential components of whole-life settlements of tolerably mobile subsea foundations through the assumption that a drained soil response is an appropriate proxy for alternating stages of undrained sliding and consolidation.

\section{Acknowledgments}

This work forms part of the activities of the Centre for Offshore Foundation Systems (COFS), currently supported as a node of the Australian Research Council's Centre of Excellence for Geotechnical Science and Engineering, and through the Fugro Chair in Geotechnics, the Lloyd's Register Foundation Chair and Centre of Excellence in Offshore Foundations and the Shell EMI Chair in Offshore Engineering.

The work was carried out while the first author was a visiting scholar at COFS, supported through the Worldwide University Network (WUN) Research Mobility Program. 
488 The second author is supported through ARC grant CE110001009 and third author through the 489 Fugro Chair in Geotechnics. The work presented in this paper was supported by ARC grant 490 DP140100684. All financial support is gratefully acknowledged.

491 The authors are grateful to Dr. Xiaowei Feng for providing the finite element analysis 492 validation of the shear stress distribution.

493

4949 Notation list

495

\section{Symbol Description}

A Geometrical distance to calculate the stress distribution beneath the foundation

$A_{d} \quad$ Flow rule multiplier

B $\quad$ Foundation width

C Geometrical distance to calculate the stress distribution beneath the foundation

D Geometrical distance to calculate the stress distribution beneath the foundation

L $\quad$ Foundation length

$b \quad$ Distance between the current stress state $\sigma$ and the conjugate one on the bounding surface $\boldsymbol{\sigma}^{\boldsymbol{B}}$

$b^{M} \quad$ Distance between the current stress state $\sigma$ and the conjugate one on the memory surface $\boldsymbol{\sigma}^{\mathbf{M}}$

$b_{\max } \quad$ Maximum value of $\mathrm{b}$

$\beta \quad$ Parameter controlling the amount of settlement in the first slide

d Dilatancy flow rule

$\delta_{v} \quad$ Vertical displacement

e $\quad$ Void ratio 


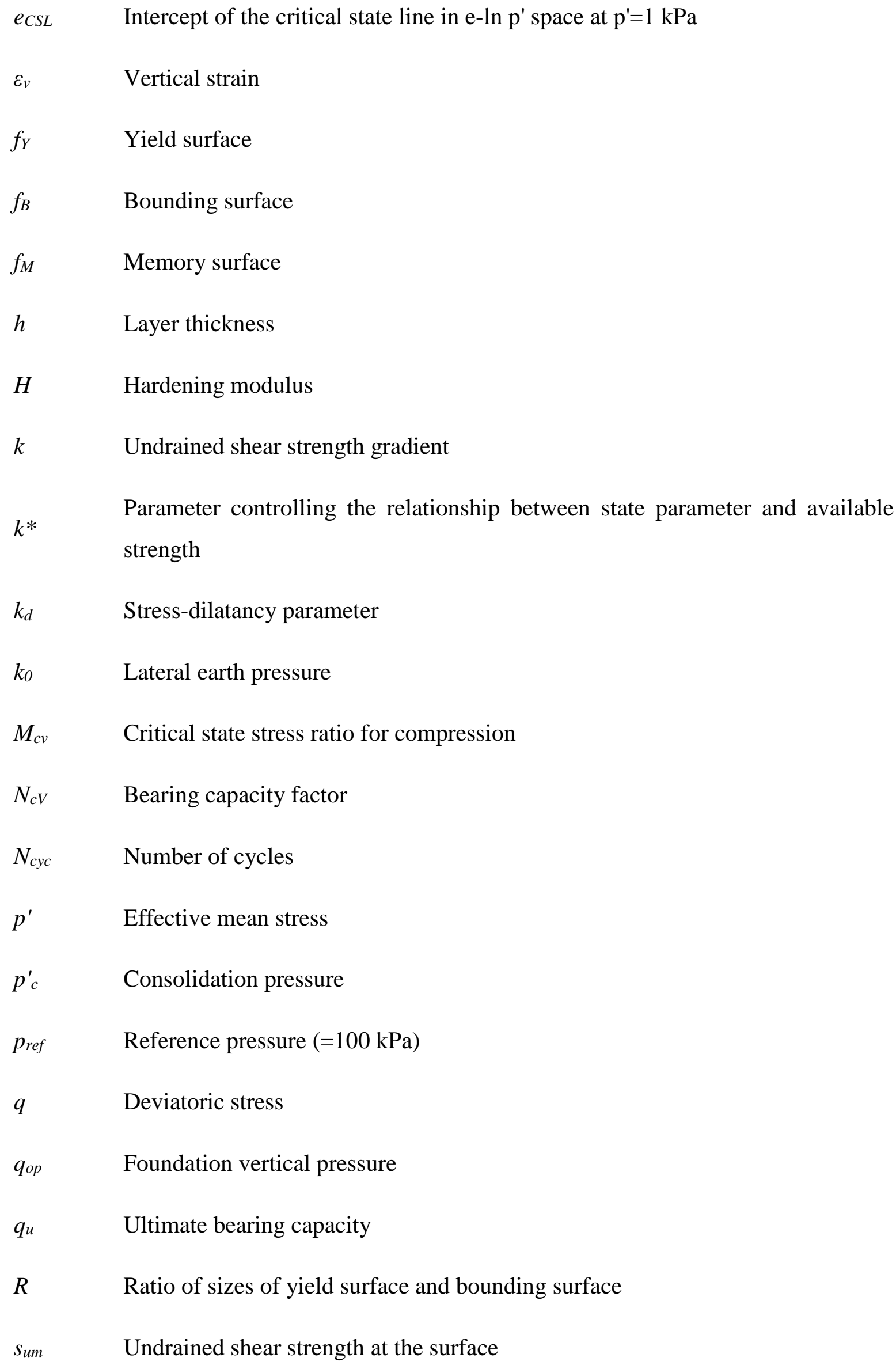




\begin{tabular}{|c|c|}
\hline$z$ & Depth \\
\hline$\gamma^{\prime}$ & Effective unit weight \\
\hline$\eta$ & Stress ratio \\
\hline$\kappa$ & Slope of re-compression line in the e-ln p' space \\
\hline$\lambda$ & Slope of the critical state line in e-ln p' space \\
\hline$\mu$ & Constitutive parameter affecting the MSH model response in cyclic conditions \\
\hline$v$ & Poisson's ratio \\
\hline$\sigma$ & Stress state \\
\hline$\sigma^{B}$ & Conjugate stress point on the bounding surface \\
\hline$\sigma^{M}$ & Conjugate stress point on the memory surface \\
\hline$\sigma_{v}^{\prime}$ & Effective vertical stress \\
\hline$\varsigma$ & Constitutive parameter affecting the contraction of the memory surface \\
\hline$\tau_{\max }$ & Maximum shear stress under cyclic loading \\
\hline$\psi$ & State parameter \\
\hline$\Delta \tau_{c y c}$ & Cyclic amplitude in each soil layer \\
\hline
\end{tabular}

\section{$498 \quad 10 \quad$ References}

499 [1] Cathie, D., Morgan, N. \& Jaeck, C. Design of sliding foundations for subsea structures. In:

500 Brown Editor. BGA International Conference on Foundation. Dundee; 2014. pp. 24-27.

501 [2] Cocjin M, Gourvenec S, White DJ, Randolph M. Tolerably mobile subsea foundations observations

of performance.

Géotechnique 2014;64(11):895-909. 
504 [3] Deeks A, Zhou H, Krisdani H, Bransby F, Watson P. Design of direct on-seabed sliding 505 foundations. In: Proceedings of the ASME 2014 33rd International Conference on Ocean, 506 Offshore and Arctic Engineering. San Francisco; 2014. p. V003T10A024. DOI: 10.1115/OMAE2014-24393

[4] Cocjin M, Gourvenec S, White DJ, Randolph M. Effects of drainage on the response of a sliding foundation. In: Meyer Editor. Frontiers in Offshore Geotechnics III. London: Taylor \& Francis Group; 2015. p.777-82. DOI: 10.13140/RG.2.1.2621.4561

[5] Roscoe KB, Burland JB. On the generalised stress-strain behaviour of 'wet clay'. In. Heyman, Leckie editors. Engineering Plasticity. Cambridge: Cambridge University Press. 1968; p. 535-609.

[6] Poulos HG, Davis EH. Elastic Solutions for Soil and Rock Mechanics. New York: John Wiley \& Sons Inc; 1974.

[7] Feng X, Gourvenec S.M. Modelling sliding resistance of tolerably mobile subsea mudmats.

[8] Gajo A, Muir Wood D. Severn-Trent sand: a kinematic-hardening constitutive model: the q-p formulation. Géotechnique 1999;49(5):595-614. DOI: 10.1680/geot.1999.49.5.595 [9] Gajo A, Muir Wood D. A kinematic hardening constitutive model for sands: the multiaxial formulation. International Journal for Numerical and Analytical Methods in Geomechanics 1999;23(9):925-65. DOI: 10.1002/(SICI)1096-9853(19990810)23:9<925::AID-

NAG19>3.0.CO;2-M

524 [10] Corti R, Diambra A, Nash DFT, Muir Wood D. An evolving memory surface for modelling the cyclic behaviour of granular soils. In: Manzanal, Sfriso, editors. Proceedings of the XV Panamerican Conference on Soil Mechanics and Geotechnical Engineer. Buenos Aires 
528 [11] Corti R, Diambra A, Escribano DE, Nash DFT, Muir Wood D. A memory surface 529 hardening model for granular soils under repeated loading conditions. Journal of Engineering 530 Mechanics 2016;142(12). DOI: 10.1061/(ASCE)EM.1943-7889.0001174

531 [12] Corti R. Hardening memory surface constitutive model for granular soils under cyclic 532 loading conditions. PhD Thesis University of Bristol; 2016.

533 [13] Roscoe KH, Schofield AN, Wroth CP. On the Yielding of Soils. Géotechnique 534 1958;8(1):22-53. DOI: 10.1680/geot.1958.8.1.22

535

[14] Muir Wood D. Soil behaviour and critical state soil mechanics. Cambridge University Press; 1990.

537

[15] Argyris JH, Faust G, Szimmat J, Warnke EP, Willam KJ. Recent developments in the finite element analysis of prestressed concrete reactor vessels. Nuclear Engineering and Design 1974;28(1):42-75. DOI: 10.1016/0029-5493(74)90088-0

[16] Been K, Jefferies M. A state parameter for sands. Géotechnique. 1985;35(1):99-112. DOI: 10.1680/geot.1985.35.2.99

[17] Dafalias YF, Popov EP. A model of nonlinearly hardening materials for complex loading. Acta Mechanica. 1975;21(3):173-92. DOI: 10.1007/BF01181053

[18] Dafalias YF. Bounding surface plasticity. Part I: Mathematical foundation and hypoplasticity. Journal of Engineering Mechanics. 1986;112(9):966-87. DOI: 10.1061/(ASCE)0733-9399(1986)

547 [19] Hardin BO, Black WL. Sand stiffness under various triaxial stresses. Journal of the Soil 548 Mechanics and Foundation Division 1966;92(SM2):27-42.

549 [20] Stewart DP. Lateral loading of piled bridge abutments due to embankment construction. PhD Thesis University of Western Australia; 1992. 
551 [21] Acosta-Martinez HE, Gourvenec SM. One-dimensional consolidation tests on kaolin clay.

552

553

554

555

556

557

558

559

560

561

562

563

564

565

566

567

568

569

570

571

572

In: Research Report GEO:06385, Centre for Offshore Foundation Systems, School of Civil and Resource Engineering, the University of Western Australia; 2006.

[22] Al-Tabbaa A. Permeability and stress-strain response of Speswhite kaolin. PhD Thesis University of Cambridge; 1987.

[23] Al-Tabbaa A, Muir Wood D. Some measurements of the permeability of kaolin. Géotechnique. 1987:37(4):499-503. DOI: 10.1680/geot.1987.37.4.499

[24] Jaky J. Pressure in silos. In: Proceedings of the 2nd International Conference on Soil Mechanics and Foundation Engineering. London; 1948. p. 103-7.

[25] Potts, D.M. \& Zdravkovic, L. Finite element analysis in geotechnical engineering - theory. London, UK: Thomas Telford.

[26] Feng X, Randolph MF, Gourvenec S, Wallerand R. Design approach for rectangular mudmats under fully three dimensional loading, Géotechnique 2014;64(1):51-63. DOI: 10.1680/geot.13.P.051

[27] Davis EH, Booker JR. The effect of increasing strength with depth on the bearing capacity of clays. Géotechnique 1973;23(4):551-63. DOI: 10.1680/geot.1973.23.4.551

[28] Lopez-Querol S, Coop MR. Drained cyclic behaviour of loose Dogs Bay sand. Géotechnique 2012;62(4):281-9. DOI: 10.1680/geot.8.P.105

[29] Escribano D. Evolution of stiffness and deformation of Hostun Sand under drained cyclic loading. PhD Thesis University of Bristol; 2014. 


\section{List of figures}

Fig. 1: Layout of the sliding foundation concept.

Fig. 2: Comparison of (a) volumetric response and (b) variation of void ratio with the number of cycles of a soil element close to the interface between the sliding mudmat and the soil ( $z / B=0.05$, with $z$ being the depth and $B$ being the width of the foundation), subjected to cyclic simple shear under constant total vertical stress by imposing either undrained loading with intervening consolidation or drained loading conditions, using the Modified Cam Clay constitutive soil model [5].

Fig. 3: Soil discretization beneath the sliding foundation.

Fig. 4: Comparison of stress distribution beneath the sliding foundation from elastic solution and finite element analysis.

Fig. 5: Representation of the adopted constitutive models in the triaxial stress space a) Modified Cam Clay (MCC) Model b) Severn-Trent Sand (STS) model c) Memory Surface Hardening (MSH) model.

Fig. 6: Calibration of the memory surface model against a drained cyclic triaxial test a) volumetric response and b) deviatoric response with the number of cycles. The test was performed under constant cell confining pressure of $300 \mathrm{kPa}$ while the initial void ratio of the specimen $e_{0}=1.08[22]$.

Fig. 7: Profile with depth of in situ a) vertical and horizontal effective stresses and b) void ratio at the end of the geostatic stage.

Fig. 8: Representation of the geometrical quantities to define the soil element state in the centreline of a foundation [6].

Fig. 9: Profiles with depth of a) normalised increase of vertical stress with depth induced by foundation operative stress, and (b) normalised tangential stress induced by foundation sliding. 
Fig. 10: Predicted normalized cumulative vertical settlements using a) MCC model b) STS model c) MSH model d) Comparison among the constitutive models.

Fig. 11: Predicted normalized vertical settlements during the first loading cycle.

Fig. 12: Shear stress-vertical strain curves for the top soil layer performed using a) MCC model b) STS model c) MSH model.

Fig. 13: Prevalent mechanisms for the adopted constitutive models a) MCC model b) STS model c) MSH model.

Fig. 14: Void ratio evolution during the test assuming a) MCC model b) STS model c) MSH model.

Fig. 15: Parametric analysis to predict the normalized cumulative vertical settlements measured by Cocjin et al. 2014 [2] using a) $\mu=11.5$ b) $\mu=8$ c) $\mu=5$ d) Comparison among the three simulations.

Fig. 16: a) Void ratio changes during the life-cycle of a sliding foundation b) Cumulative vertical displacements with depth at the stable state.

Fig. 17: Prediction of life-cycle settlements of sliding foundations a) on soils of different undrained shear strength profiles b) for sliding foundations with different aspect ratios $B / L$. 


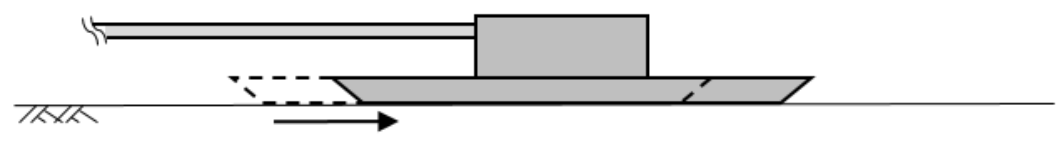

Figure 1
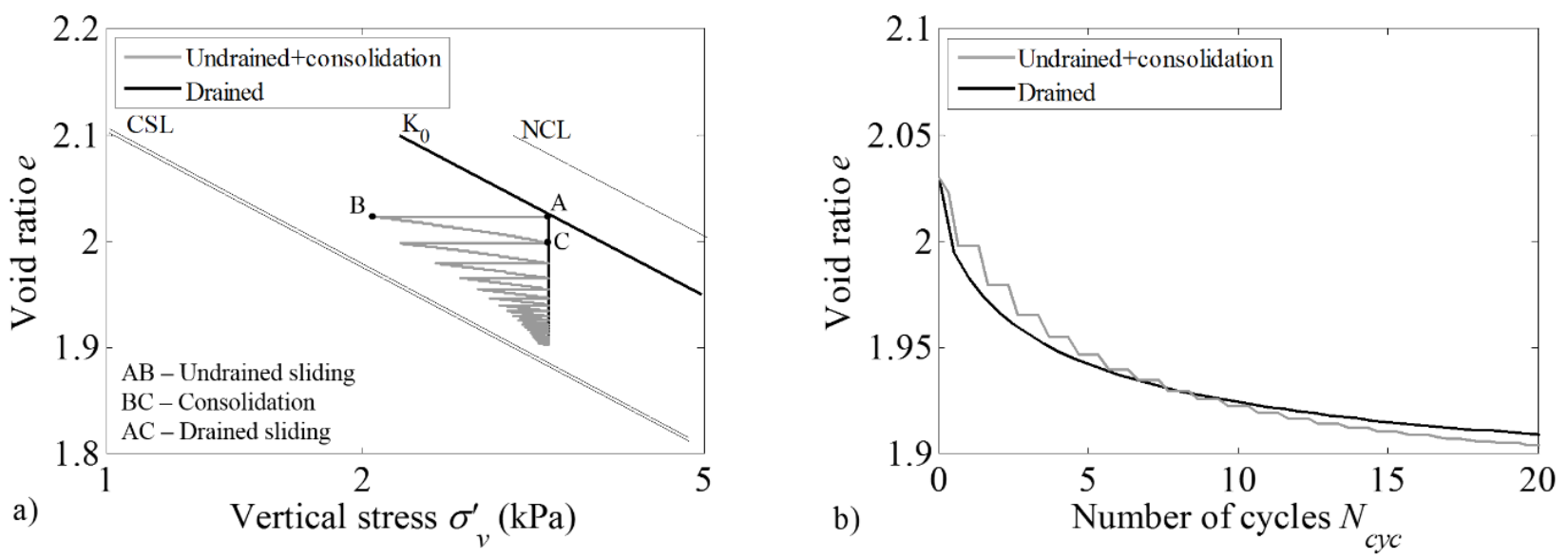

Figure 2 


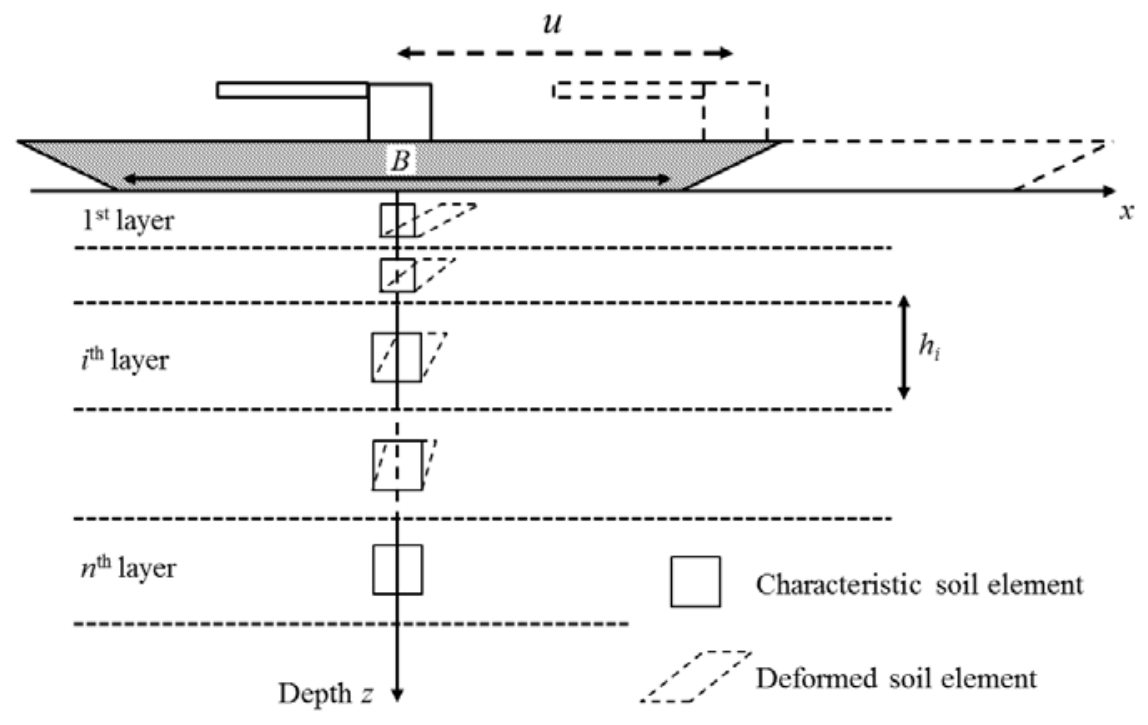

Figure 3

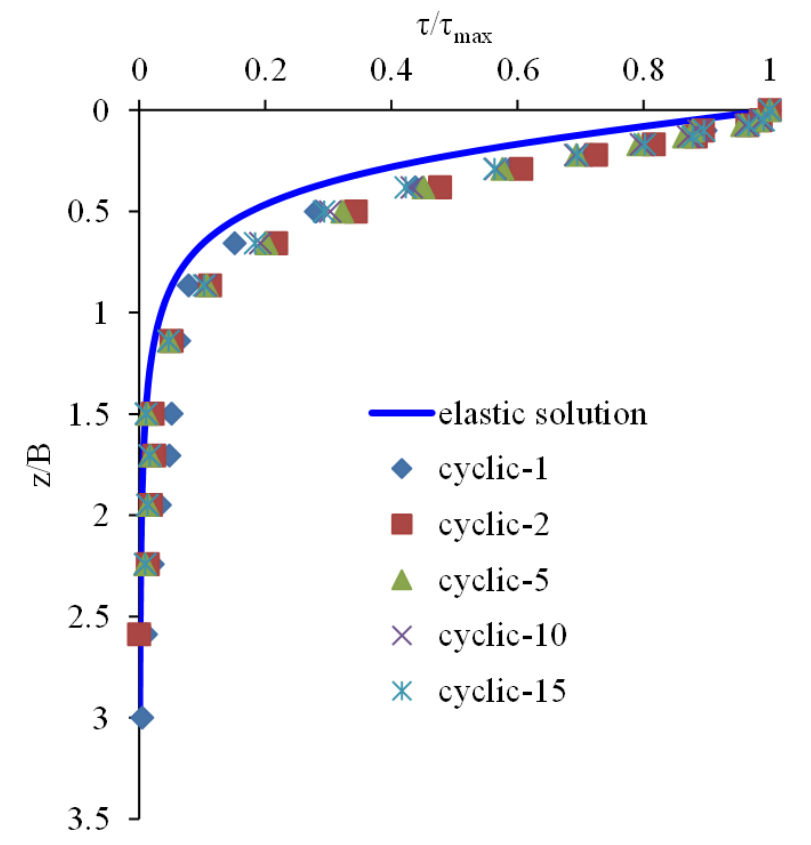

Figure 4 
MODIFIED CAM CLAY (MCC)

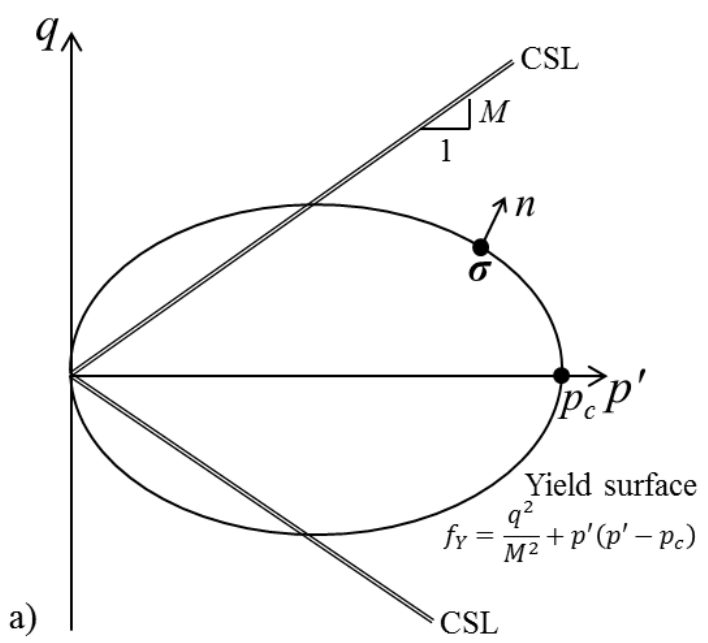

SEVERN-TRENT SAND (STS)

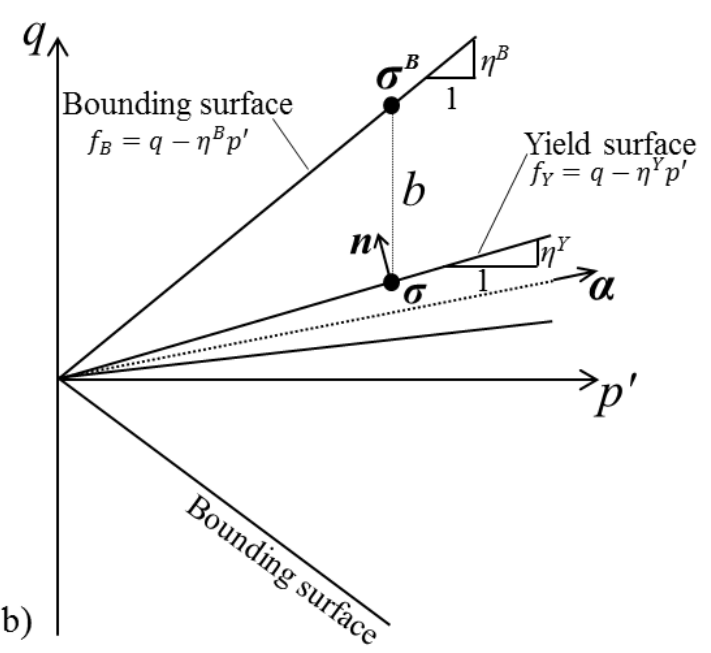

MEMORY SURFACE HARDENING (MSH)

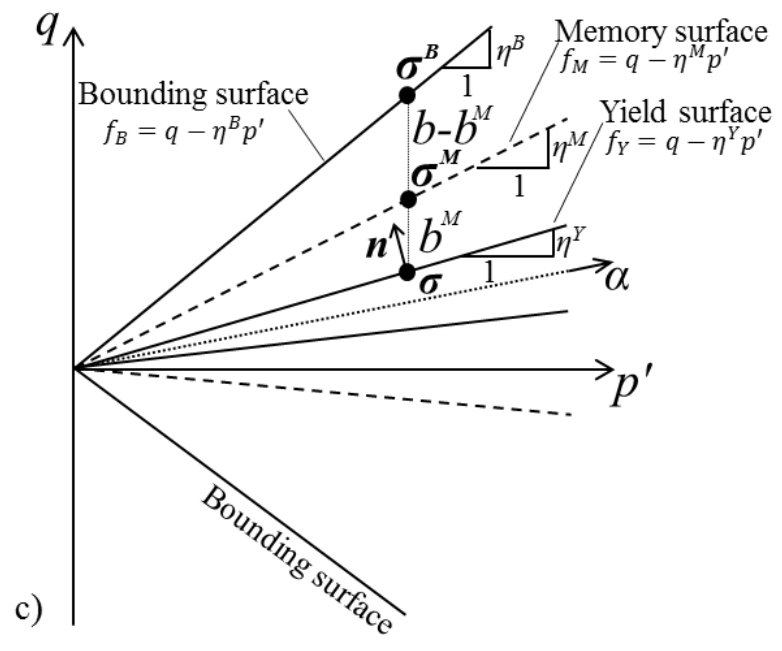

Figure 5 

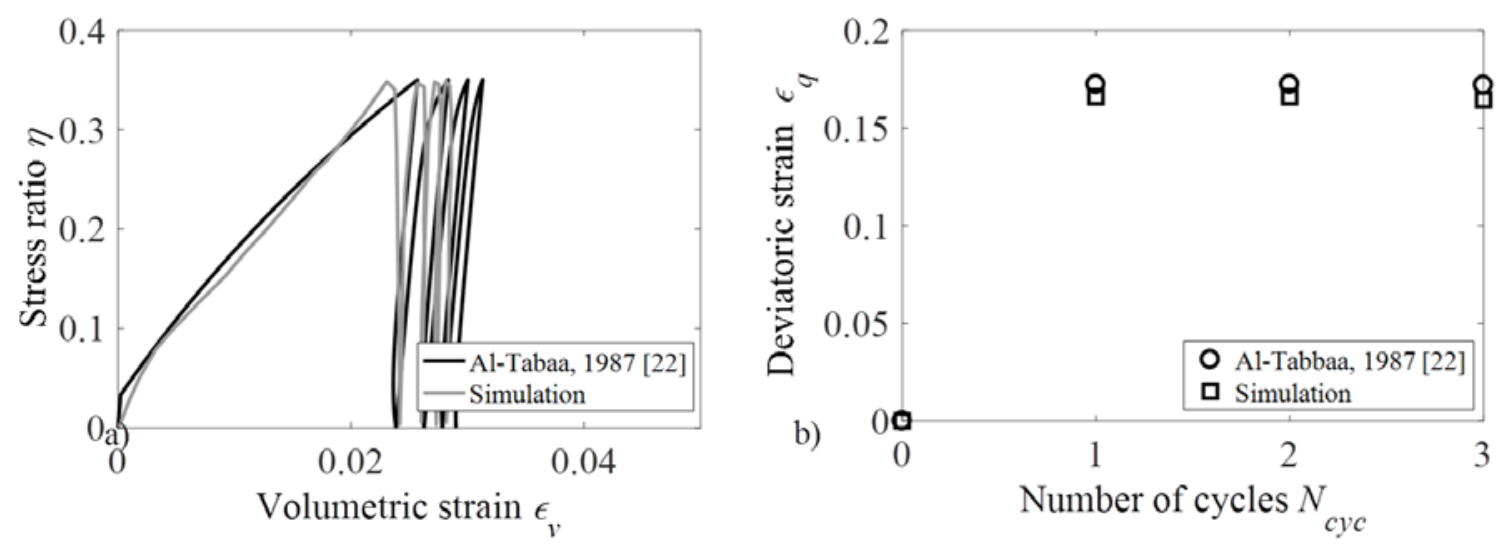

Figure 6
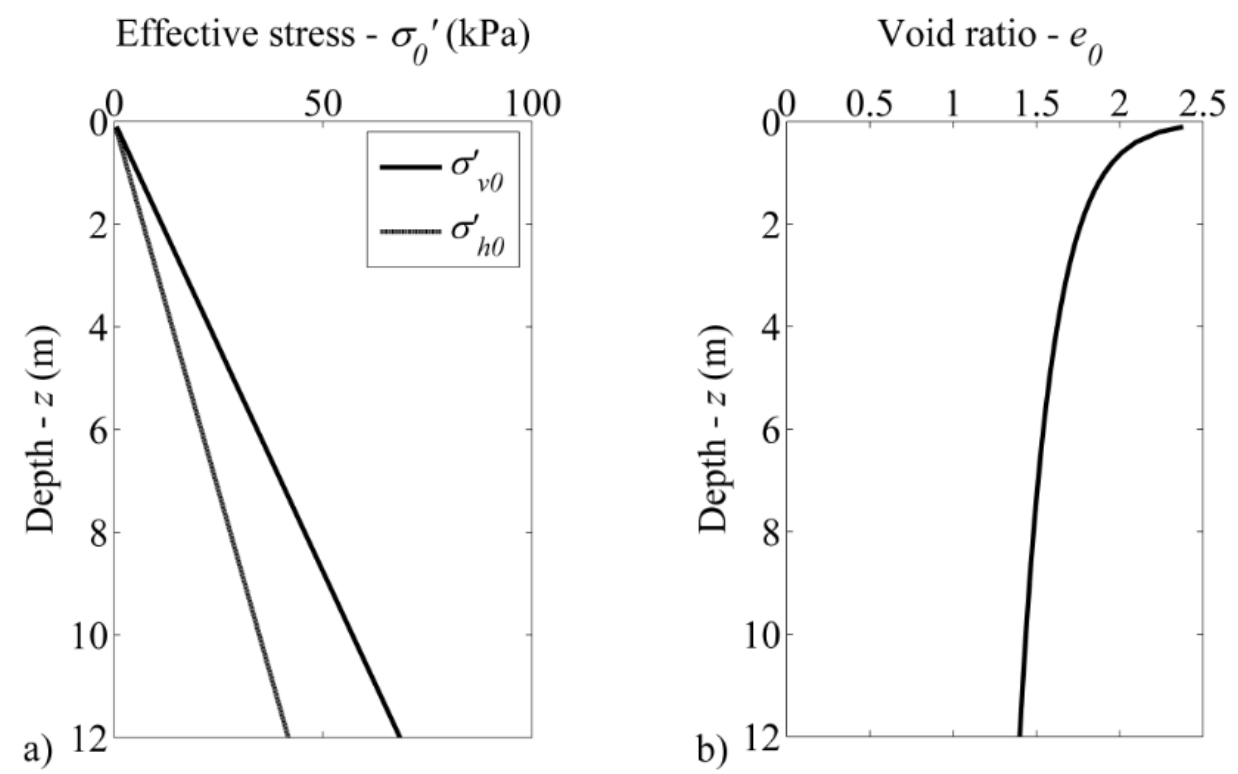

Figure 7 


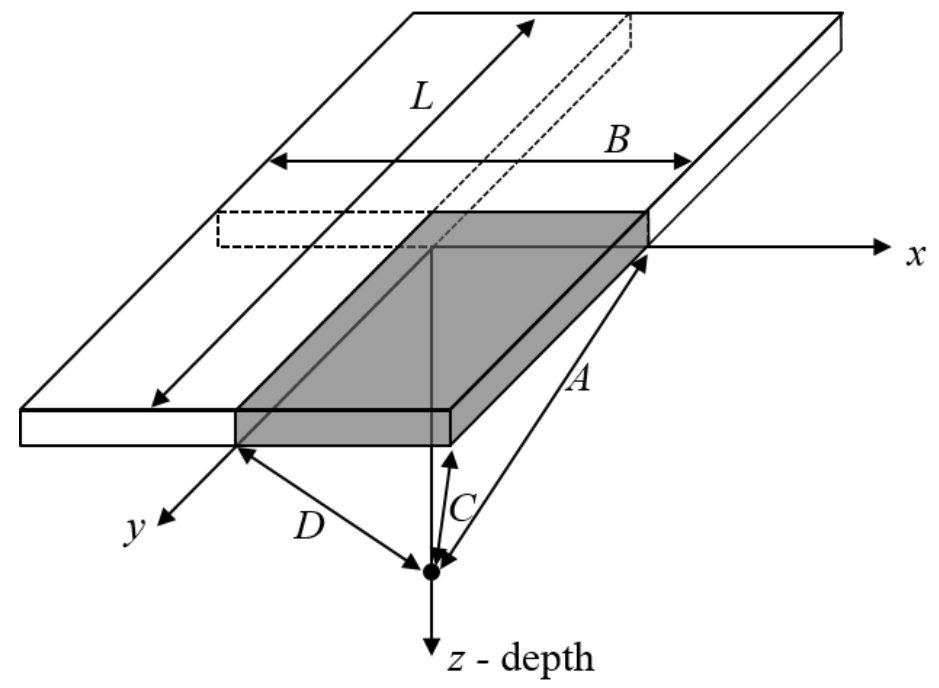

Figure 8
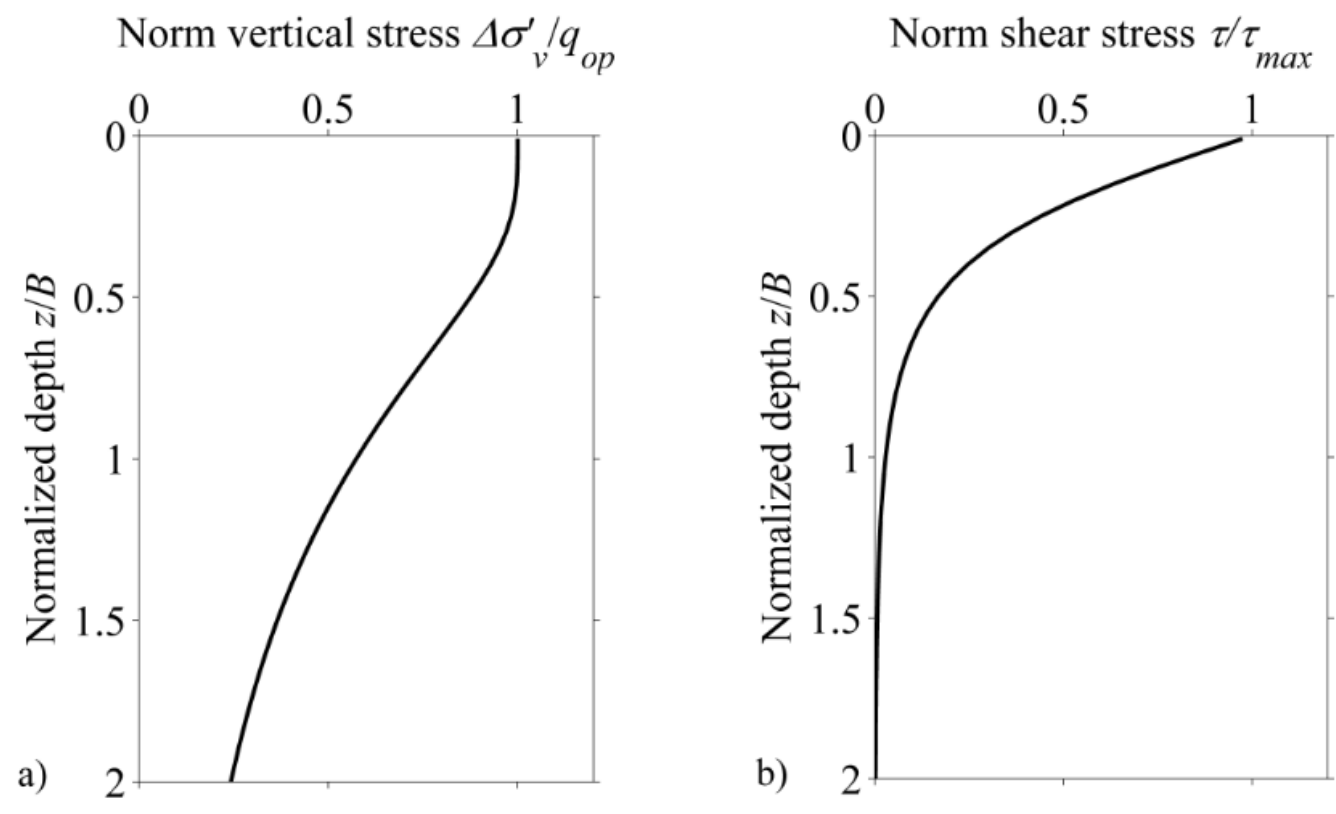

Figure 9 


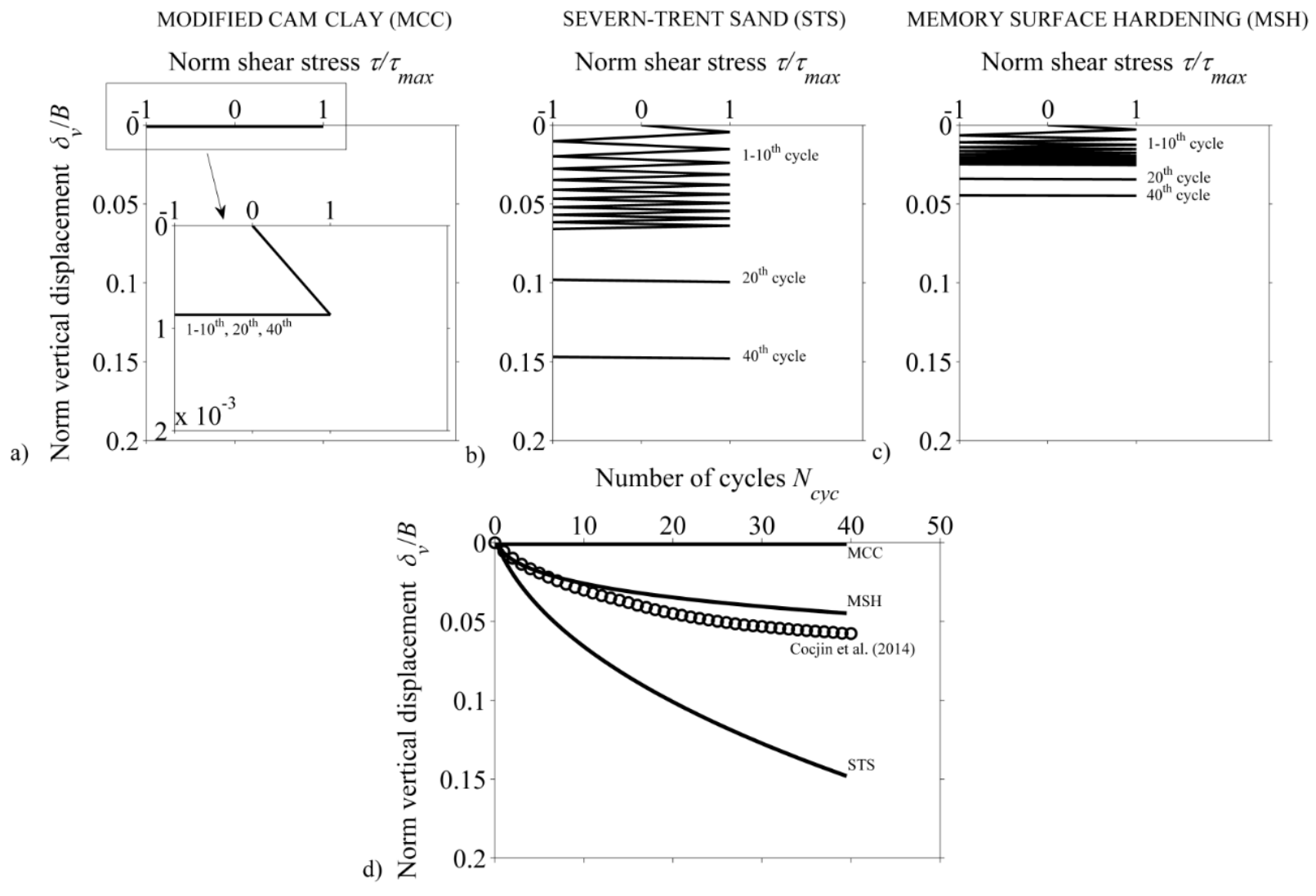

Figure 10

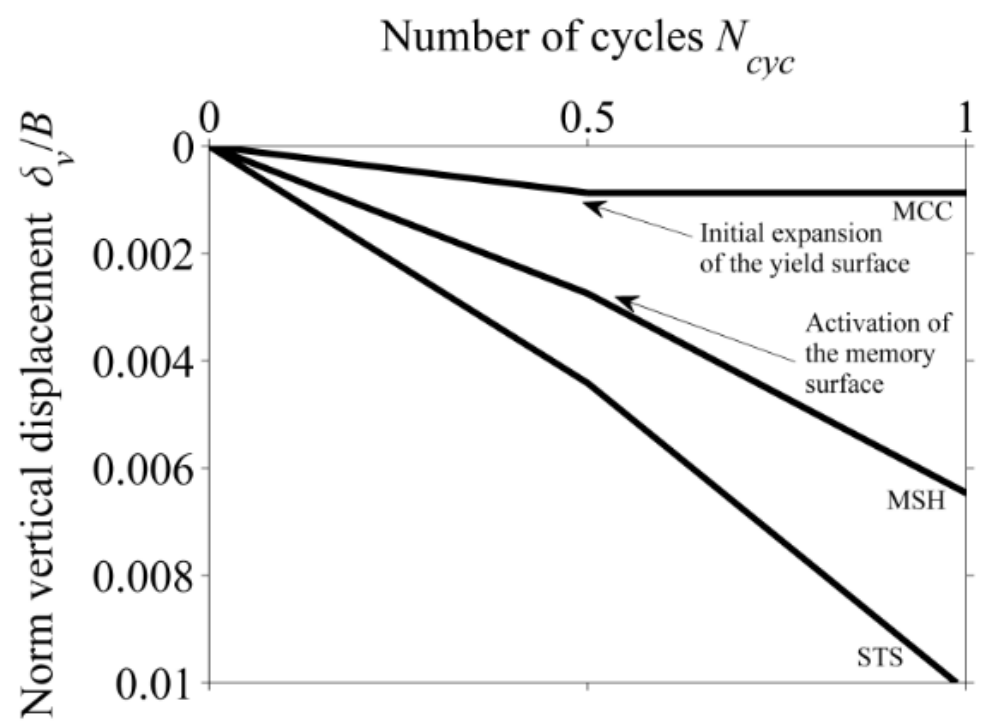

Figure 11 

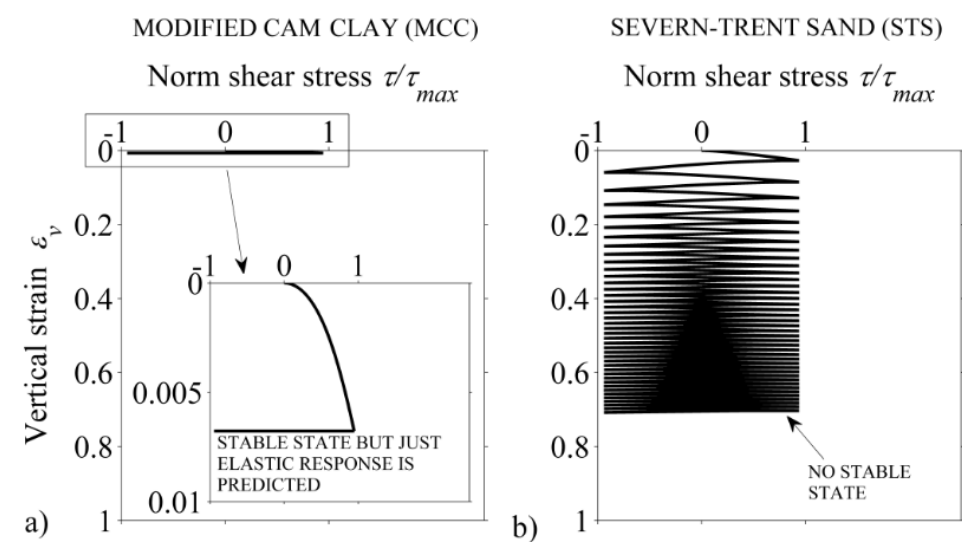
MEMORY SURFACE HARDENING (MSH) Norm shear stress $\tau / \tau_{\max }$

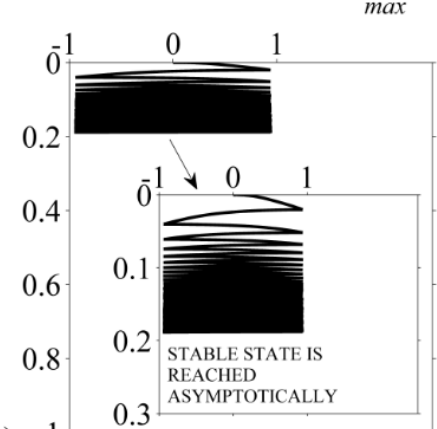

Figure 12

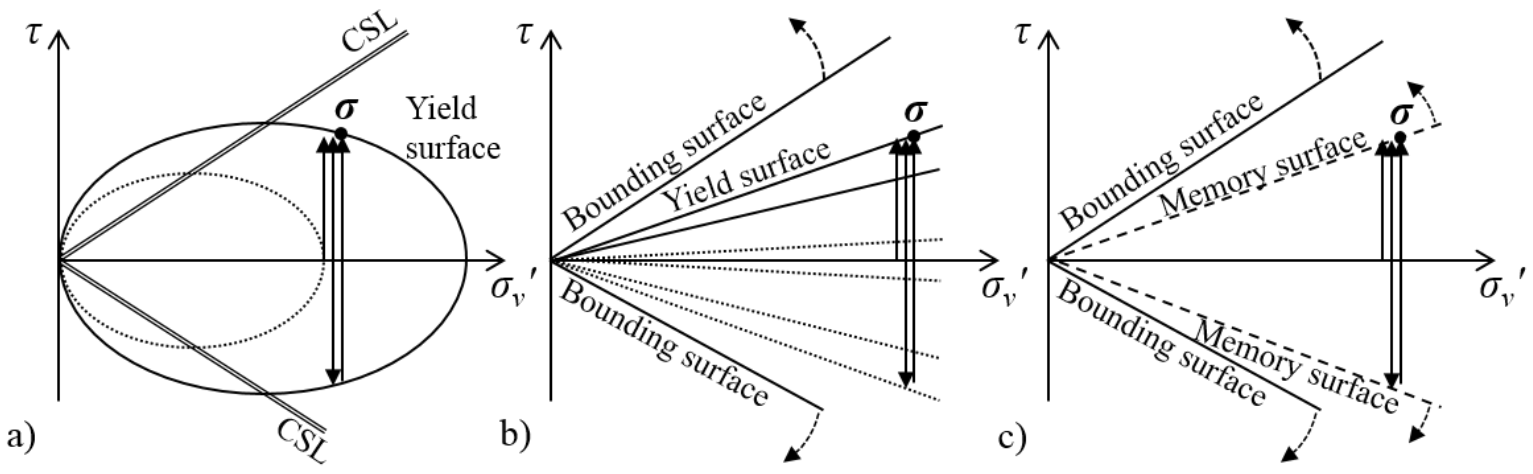

Figure 13 


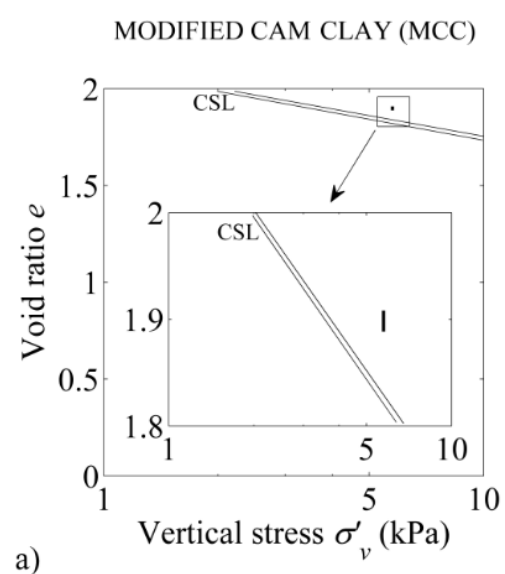

a)
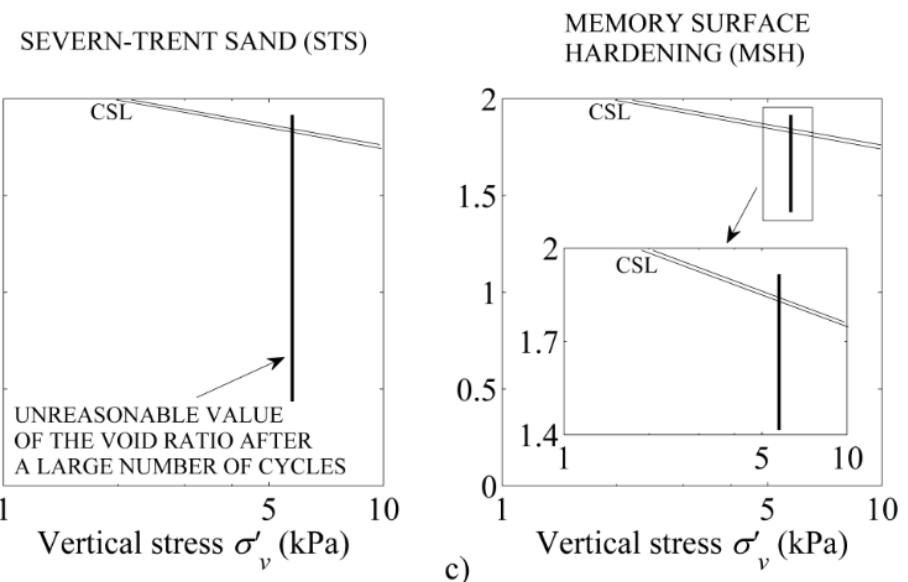

Figure 14

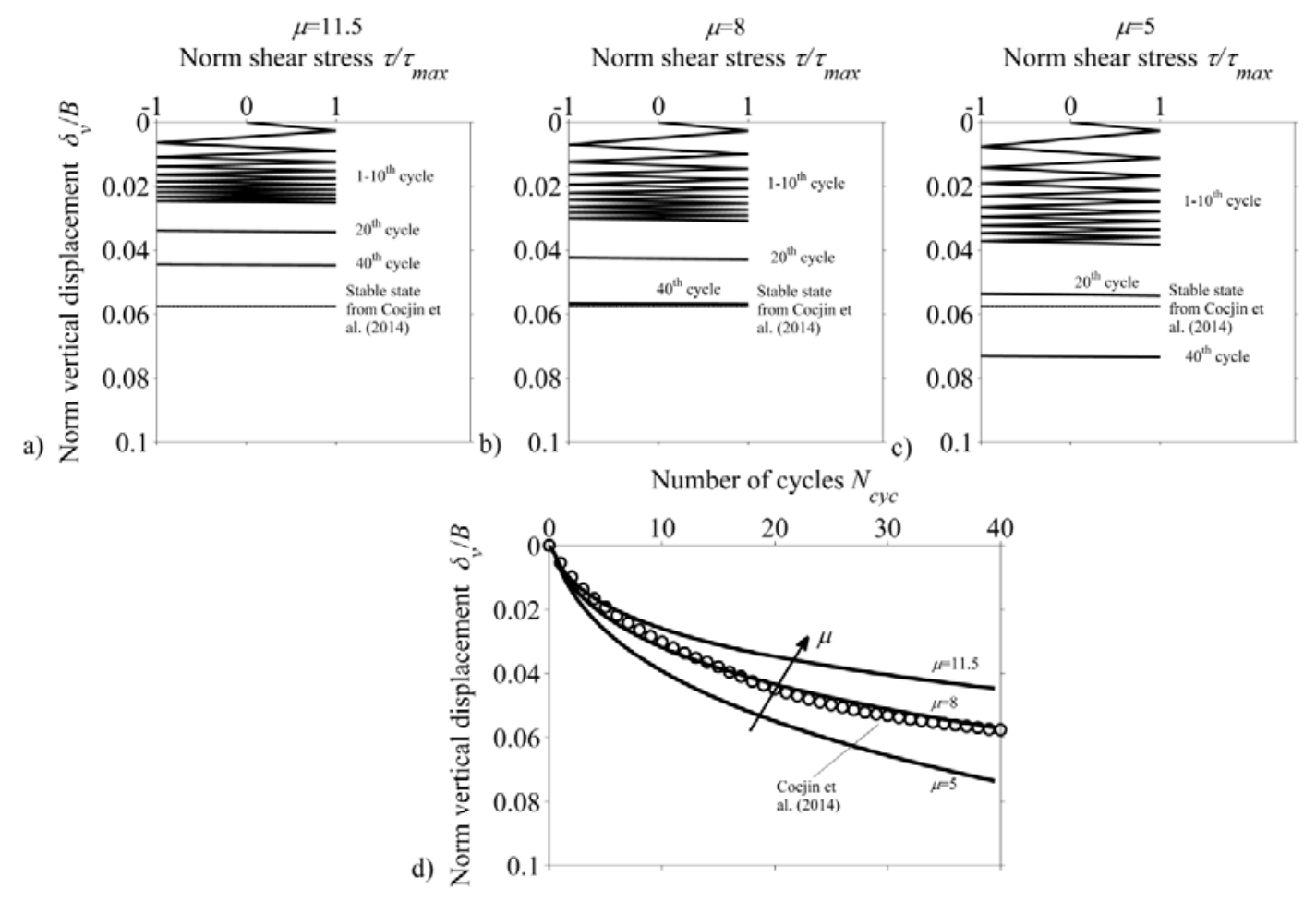

Figure 15 

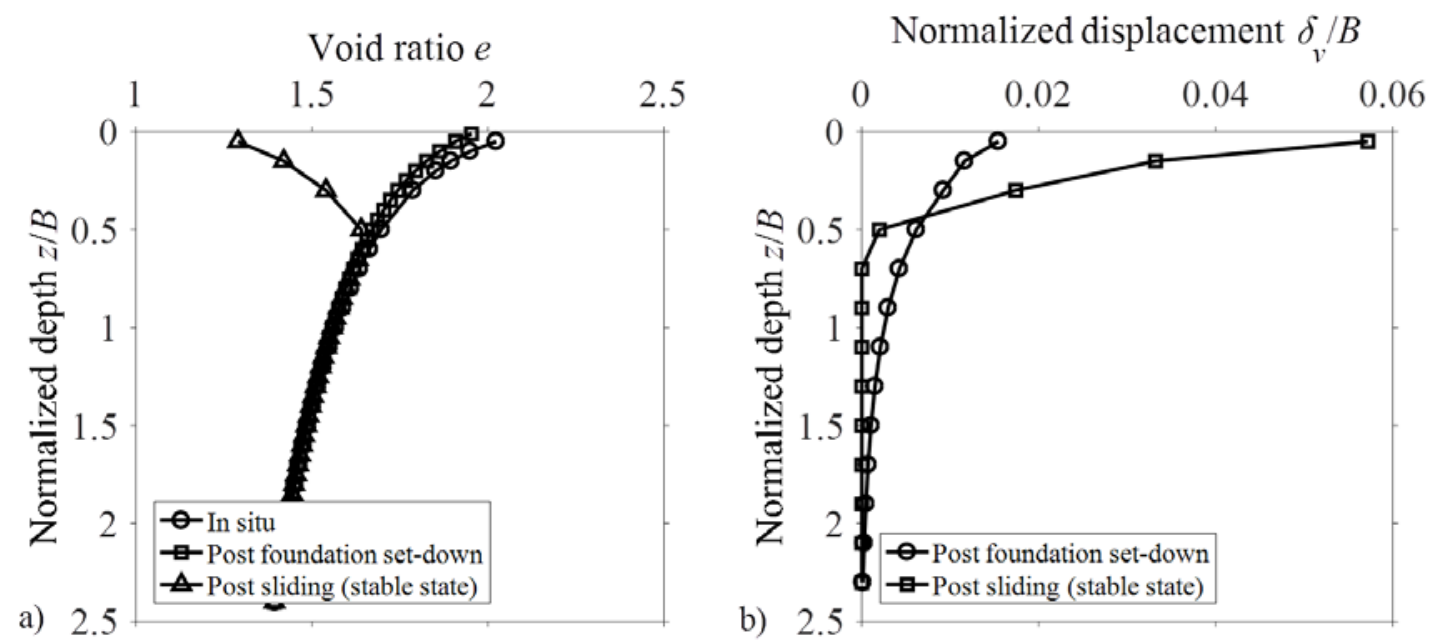

Figure 16
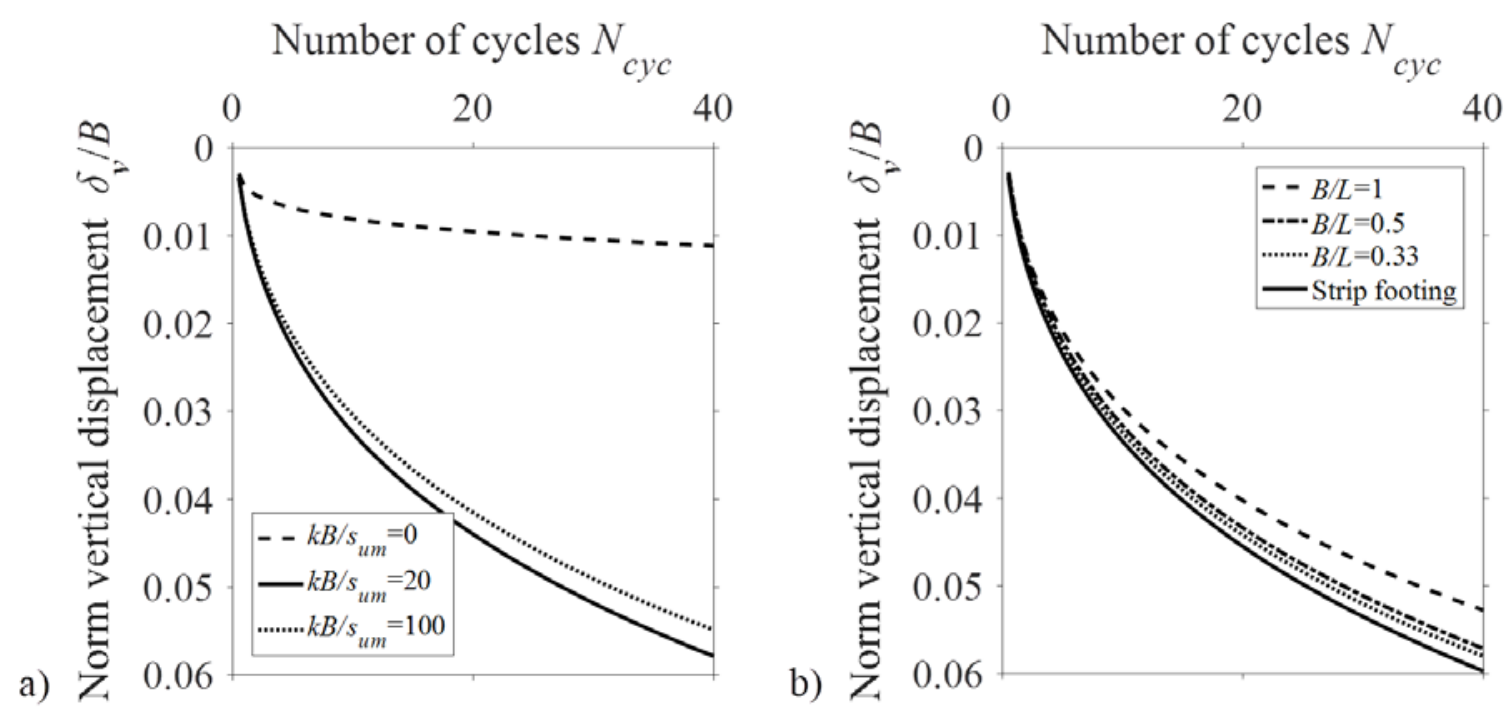

Figure 17 